\title{
GUNK, TOPOLOGY AND MEASURE
}

\author{
Frank Arntzenius
}

arntzeni@rci.rutgers.edu

\section{Introduction}

It is standardly assumed that space and time consist of extensionless points. It is also a fairly standard assumption that all matter in the universe has point-sized parts. We are not often explicitly reminded of these very basic assumptions. But they are there. For instance, one standardly assumes that one can represent the states of material objects, and of fields, by functions from points in space and time to the relevant point values. Electric fields, mass densities, gravitational potentials, etc.... are standardly represented as functions from points in space and time to point values. This practice would seem to make no sense if time and space did not have points as parts.

There is an alternative that has not been much explored. The alternative is that space and time and matter are 'pointless', or 'gunky'. The idea here is not that space and time and matter have smallest finite-sized bits, that space and time and matter are 'chunky'. Rather the idea is that every part of space and time and matter has a non-zero, finite, size, and yet every such part can always be subdivided into further, smaller, parts. That is to say, the idea is that every part of space and time and matter has a non-zero size, and yet there is no smallest size.

Let me emphasize how radical this idea is. It is very natural to think that any thing decomposes into some ultimate collection of fundamental parts. And it is very natural to think that the features of any object are determined by the way that object is constructed from its ultimate parts, and by the elementary features of these ultimate parts. Indeed, much of the history of science can be seen as an attempt to break down complex objects and processes into ultimate parts, and to find the laws that govern these ultimate parts. But if there are no smallest regions, and if there are no smallest parts of objects, then a spatial or temporal decomposition of a region, and of an object, can not bottom out at an ultimate level. The idea that the features of large regions and large objects are determined by the features of minimal-sized regions and minimalsized objects can not work if space and time, and the objects in it, are gunky, i.e. pointless. Space, time, and objects would simply not have ultimate parts. There would just be an infinite 
descending chain of ever-smaller parts. A somewhat dizzying prospect.

Well, let's not get ahead of ourselves. Not only would it require a fairly radical revision of our atomistic intuitions, it would also require a fairly radical and extensive re-working of standard mathematical methods for doing physics. If we can not use real numbers to coordinatize locations in space and time, what can we use? If we can not use ordinary functions to describe the states of things, what can we use?

All things in good order. We will get started on the business of re-writing physics a bit later on. First we will consider arguments for undertaking this seemingly mad enterprise. To preview: we will find no utterly compelling arguments against the existence of points. But we will find non-compelling reasons to explore the mathematics of gunky space and time.

\section{The possibility of motion and determinism}

Zeno argued that if time consists of instants of zero duration, then during each such instant an object can not move. But if time consists entirely of a series of such instants then objects can never move. In view of this problem Aristotle proposed that there are no instants, no 0 -sized intervals of time, indeed no smallest sized, atomic, intervals of time. Rather, time consists of smaller and smaller intervals. To put it another way: the world is a true movie, not a sequence of snapshots. To put it even more suggestively: becoming is not reducible to being.

One may not be impressed by Zeno’s argument. One may for instance respond, as did some commentators in the middle ages, that motion is just to be at different locations at different times, and that it therefore simply is not true that just because one occupies only one location at one time, that one therefore never moves.

Indeed, this is a perfectly coherent way to respond to Zeno's problem. But one can then formulate a new worry, which is closely related to Zeno's worry. For if motion is just a matter of being at different locations at different times, then the intrinsic state of an object at an instant does not include its velocity. How then does an object at an instant 'know' in which direction to continue and at which speed? Less anthropomorphically: if the instantaneous states of objects do not include their velocities, then how could the instantaneous state of the world determine its subsequent states? I.e. how could determinism hold? The world may in fact develop in a deterministic fashion, and it may not, but surely whether it does, or does not, should depend on 
the character of the laws of evolution of the world, rather than that the atomicity of the structure of time alone should imply that the world can not be deterministic. ${ }^{1}$

One might attempt to respond to this argument by claiming that even if time consists of 0 -sized instants, nonetheless the intrinsic state of an object at a time does include a velocity. Such an 'intrinsic velocity' would not be defined (as in ordinary calculus) in terms of (limits of) the position development of an object. Rather, it would be a primitive intrinsic feature of an object at a time, which causes the object to subsequently move in the direction in which the intrinsic velocity is pointing.

This does not strike me as a plausible response. For according to this response the intrinsic velocity at an instant and the direction in which the trajectory in space continues are not definitionally related, but are merely causally related. So it should be logically possible to have an object whose spatial trajectory continues in a direction that differs from the direction in which its intrinsic velocity points. Now, one might claim that such a bizarre non-alignment of direction of trajectory and intrinsic velocity is ruled out by the laws of nature. However, the mere conceptual possibility of such a misalignment seems puzzling, to say the least. Furthermore if the laws of nature are to connect the directions in which primitive velocities point and the directions of trajectories in space, as they must do, then there is going to have to exist some further primitive relation, 'parallelhood', which obtains, or fails to obtain, between intrinsic velocities and spatial directions. Other things being equal it seems undesirable to add 'intrinsic velocities' and 'parallelhood' to one's stock of primitive quantities and relations, when one has no real need for them. In short, this response on behalf of points does not seem plausible to me. (For more detail on this line of argumentation, see Arntzenius 2000.)

However, a more plausible response to Zeno can be made on behalf of points. For one could simply claim that determinism should not be understood as the idea that the state at an instant determines states at all other times. Rather it should be understood as the idea that any finite history of states determines states at all other times.

${ }^{1}$ Well, it could still be deterministic if the equations of motion were first order, as they are in quantum mechanics. Still, one might like to think that even if the equations of motion are second order, as they are in classical mechanics, the world could be deterministic. 
Indeed, it seems to me that Zeno's arrow provides no compelling argument against pointsized instants. Let's turn to another argument.

\section{Cutting things in half}

If space consists of points then one can not cut a region exactly in two halves. For if one of the two regions includes the point on the cutting line, i.e. if it is closed at the cut, then the other does not include the points on the cutting line, i.e. it is open at the cut. Imagine, for instance, that we have $\mathrm{x}$ and $\mathrm{y}$ coordinates which are parallel to the sides of a rectangle. Suppose that the horizontal, $\mathrm{x}$-coordinate, of the rectangle runs from 0 to 2, and suppose that we cut the rectangle at $x=1$. The question then arises: do the points that have $x$-coordinate $=1$ belong to the left hand side after we have made our cut, or to the right hand side? If they belong to the left hand side, then the left half is closed at the cut, and the right half is open. If vice versa, then the left side is open. So the two parts would not be identical. So one can not cut a region exactly in half if regions are composed out of points. One might reasonably conjecture that such a difference between open and closed regions is an artefact of our mathematical representation of regions, that does not correspond to a difference in reality. I, for one, find it hard to believe that there really are distinctions between open and closed regions in nature. But I agree that this is hardly a knock-down argument.

\section{Paradoxes of size}

If there exist points in space, and space is continuous, then it can be shown that there must be regions that have no well-defined size. For instance, there will be a part of any wall in any room such that it has no well-defined size. If you wanted to paint such a part of a wall in your house blue, there would be no possible answer to the question: "How much paint will I need to paint that part of my wall blue?". The problem is not that you would not know how much paint you would need, or that you would need 0 quarts of paint. Rather, the problem is that there just exists no quantity $r$ of paint such that you would need exactly that quantity to paint that region. Let me be a bit more precise.

One can prove that in a continuous, pointy space there must exist regions that have no well-defined measure, if one assumes the axiom of choice and one assumes that the measure is 
countably additive. One can also prove that in a continuous pointy space of 3 or more dimensions there must exist regions that have no well-defined measure, if one assumes the axiom of choice and one assumes that the measure is finitely additive and invariant under (distance preserving) translations and rotations. ${ }^{2}$

There is even more weirdness about points and sizes: Banach and Tarski have shown that the existence of points implies cost-free guaranteed increases in size. That is to say, they showed that in a continuous pointy 3 -dimensional space one can take a sphere, break it into a finite number of pieces (five pieces in fact), move those pieces around rigidly (i.e. while preserving distances between the parts of the pieces), and re-arrange those pieces to form a sphere of exactly twice the size! That is to say, by breaking an object into five parts, and merely rearranging these parts spatially, without any stretching or changing of shapes, one can make an object larger or smaller, as one desires.

There is in fact a close relationship between this result and the fact that there are regions which have no well-defined size. Some of the parts into which we must break the sphere must have no well-defined sized. It is not hard to see that this must be so, for rigid motions preserve size, and the size of an object that consists exactly of five non-overlapping parts is just the sum of the sizes of those parts. So Banach and Tarski's result depends essentially on the existence of size-less regions.

How might one respond on behalf of points? Well, in the first place, one might simply deny the axiom of choice. This is an issue that could take us deep into philosophy of mathematics and mathematical physics, to which I have nothing new to contribute. I merely wish to point out that denying the axiom of choice implicitly commits one to (being part of) a large project, namely that of re-writing that part of mathematics and mathematical physics that one wants to retain in a manner that makes no use of the axiom of choice. All I can say in response is that I am interested in a different project, namely that of doing physics without points. My project has several independent motivations, only one of which concerns the measure theoretic paradoxes.

A second possible response to the measure theoretic paradoxes is: who cares? Surely we

${ }^{2}$ See e.g. Skyrms (1983) and Wagon (1985). 
will not as a practical matter get our hands on measureless parts of objects. Surely size-altering de-compositions and re-compositions are not practically achievable. So why worry?

Indeed, since one needs the axiom of choice to prove the existence of measureless regions, one can not have explicit constructions of measureless, or of measure-altering, decompositions and re-compositions. Nonetheless the mere existence of regions and/or parts which have no measure, and the mere possibility of size-altering de-compositions and re-compositions, remains rather bizarre, and prima facie implausible.

A third possible response to the measure theoretic paradoxes (on behalf of points) starts by making a distinction between sets of points in space-time, which are mathematical entities, and physical regions. One could, e.g., suggest that all physical regions are Borel regions. ${ }^{3}$ If that is so, then all physical regions are (Lebesque) measurable, and no size-altering de-compositions and re-compositions are possible.

Indeed, one could say this. But note that this means that regions fail to satisfy the standard axioms of mereology. For one is denying that the fusion of any arbitrary collection of regions is a region. (Some collections of points are such that their fusion is a non-Borel region.) It seems hard to motivate this failure independently.

Nonetheless, yet again, we have found no devastating argument against points. We have simply found one more reason to try to see how far we can go without points. Let us turn to another argument against points.

\section{Quantum mechanics and points}

In non-relativistic quantum mechanics one can represent the state of a single particle by a wavefunction. The probability that a particle will be found in a particular region upon measurement is given by the integral of the square of this wave-function in this region. If one has two functions whose values differ on a set of points of measure 0 , then integrating them over any region will always yield identical results. Thus, as far as probabilities of results of measurements are concerned functions that differ on a set of points of measure 0 are equivalent. This provides

${ }^{3}$ Borel regions of the real line: start with the collection of all open intervals, then close this collection up under countable union and intersection, and complementation. 
motivation for the claim that functions that differ on a set of points of measure 0 correspond to the same wave-function, i.e. the same quantum state.

A slightly more formal motivation for this derives from the fact that in a Hilbert space there is a unique null vector, a unique vector whose inner product with itself is 0 . Thus, if one wishes to represent vectors in a separable Hilbert space (with a countable infinity of dimensions) by (complex) functions on space (or configuration space), and one wishes to represent the inner product of vectors by integration of the corresponding functions, then one has to represent vectors not by functions but by equivalence classes of functions whose values differ on up to (Lebesque) measure 0 points. Indeed, although it is not often brought to the fore, it is a standard assumption in quantum mechanics that wave-functions correspond to equivalence classes of (square integrable) functions that differ up to Lebesque measure 0.

This ignoring of measure 0 differences between regions in space suggests that quantum mechanics should be set in a gunky space, not in a pointy space. (We will flesh out this claim in more detail when we examine the measure theoretic approach to gunk.) But, as always, there are responses possible on behalf of the point lover.

In the first place one might respond that the above is a false claim: quantum mechanics standardly uses wave-functions that are eigenfunctions of position, so-called 'delta functions', which differ from each other only on measure 0 sets of points. This line of response takes us into a tricky area. So-called 'delta functions' are not functions at all. Indeed position operators, on the standard, separable Hilbert space, approach to quantum mechanics, simply can not have eigenstates. Nonetheless, it is true that there are (non-standard) ways of rigorizing the notion of an eigenstate of position, and thereby sanctioning states that in a clear sense are confined to a single point, while departing from the standard formalism of separable Hilbert spaces. (See e.g. Böhm 1978 and Halvorson 2001.) Not only does one have to depart from the standard formalism of separable Hilbert spaces in order to do so, but position eigenstates also have the feature that observables such as momentum and energy have no well-defined expectation values in such position eigenstates. In Arntzenius (2004) I have discussed whether it is worth paying this price for the acquisition of position eigenstates, and argued for a cautious 'no'. Let me here merely say that it is far from clear that it is worth paying this price, and leave it at that.

There is of course another possible response that can be made on behalf of the point 
lover. One could simply accept that quantum mechanics happens not to make use of measure 0 differences, and argue that this is all good and well, but this does not mean that such differences do not exist. Not every theory needs to make use of all the features that nature has on offer.

Indeed, I agree that both of the above two responses (on behalf of points) are perfectly coherent and possible. Nonetheless it seems to me that nature is piling up the hints that there just might be no points out there in space and time. Let's look at one more problem with points.

\section{Contact between objects}

In the $19^{\text {th }}$ century some people started worrying about the possibility of contact between solid objects if space consists of points. Here is a sketch of such worries. Let us suppose that solid objects can not interpenetrate, i.e. that solid objects can not occupy overlapping regions. Now consider two solid objects which always occupy closed regions, i.e. regions which include their own boundary. Such objects can never be in contact, for closed regions either overlap or are a finite distance apart. In order to avoid interpenetration such objects must decrease their velocities when they are still a finite distance apart, so some kind of action at a distance would have to occur. It seems strange and objectionable that the mere existence of solid objects should imply action at a distance. Alternatively suppose that solid objects occupy open regions. Then there must always be at least one point separating them. So they still can not be in genuine contact, and they still must change their velocities without ever being in genuine contact.

The impossibility of genuine contact seems to provide an objection to the existence of points. However, there are a number of decent responses that one can give on behalf of points.

In the first place, one could respond that one would not want such 'genuine contact' anyhow, since collisions would lead to sharp, undifferentiable, kinks in the trajectories of objects. One could plausibly argue that a more realistic physics has objects interacting through fields. Then there will never be 'genuine contact', so there is no 'problem of contact'. One could amplify this line of thought by claiming that it is even more realistic to suppose that quantum mechanics, with an ontology of wave-functions (or perhaps wave-functions plus point particles), is correct, and that given such an ontology there is no problem of contact.

Secondly, one could argue that even if one wants to countenance solid objects which interact by contact, one could just have a slightly different account of what it is to 'be in contact' 
and what it is to 'interpenetrate'. One could, e.g., just say that two objects are 'in contact' if and only if the boundaries of the regions that they occupy overlap. (A point $p$ lies on the boundary of region $R$ if any open set containing $p$ intersects both $R$ and the complement of $R$.) And one can say that objects do not 'interpenetrate' unless they overlap on more than just their boundaries. Physics can then proceed as usual. Of course, this would mean that objects occupying open regions (in a 3-dimensional space) that are separated by a two-dimensional surface are in contact, and that bodies occupying closed regions which overlap on a two-dimensional surface do not interpenetrate. But so what. It does not lead to any trouble in formulating physics, or any trouble with experiment. It only leads to trouble with philosophers who think that it is a priori that 'genuine contact' is possible, where 'genuine contact' means having not even a single point in between, and who think it is a priori that 'interpenetration' is not possible for solid objects, where 'interpenetration' means not overlapping even on a single point. I don't know whether to respond to such philosophers that in a Newtonian collision world there are, in their sense of 'solid', no solid objects, or whether to respond that in their sense of 'genuine contact' there is no genuine contact, and in their sense of 'interpenetration' there is interpenetration. But one can do Newtonian collision physics when one defines contact as having overlapping boundaries, and interpenetration as overlapping on more than a boundary.

Both of the above responses on behalf of points seem adequate. Nonethless note that neither of the responses requires a physics that makes essential use of points, or of measure 0 differences. So one is still left with the suspicion that points, and measure 0 differences, are artifacts of the mathematics, and do not exist in reality.

\section{Now what?}

It appears that every problem associated with the existence of points can be overcome; there appears to be no single devastating argument that space and time (or matter) have to be gunky. Nonetheless it remains of interest to examine the possibility of doing physics in gunky space and time in more detail.

There have been a number of approaches to the mathematics of gunky spaces. These approaches divide into three categories: the measure theoretic approach (see Skyrms 1993 and Sikorski 1964), the topological approach (see Roeper 1997), and the metric approach (see 
Gerla 1990). In this paper I will not look in any detail at the metric approach. The reason I will not is that in the metric approach one assumes from the start as fundamental notions the notion of the 'diameter' of a region and the notion of the 'distance' between regions. This approach is prima facie ill-suited for the purposes of modern physics since in general relativity the notion of distance is local and path-dependent rather, it is not a non-local path-independent relation between regions. It would seem preferable to first be able to build a pointless differentiable manifold, and then to be able to put a metric tensor field on such a differentiable manifold. The measure-theoretic and topological approach to gunk are prima facie more amenable to this idea, since they do not start by presupposing the existence of non-local metric structure. Let's look at these two approaches in more detail and let's start with the topological approach.

\section{The topological approach to pointless spaces}

My strategy for constructing a pointless topological space will be as follows. I will start with an ordinary pointy topological space. I will then put on blurry spectacles which wash out differences in regions which, intuitively speaking, are differences in the (pointy) mathematical representation of space that do not correspond to differences in actual physical space. This will yield a pointless topology. Once I have a pointless topology, I, of course, no longer have ordinary (point to point) functions. But there are still maps from pointless regions to pointless regions. We will see that a rather natural set of such maps corresponds 1-1 to pointy functions that map regular closed region to regular closed regions. Unfortunately this does not include functions which are constant on a finite region, so that we do not appear to have enough materials to do physics with. Furthermore, one would like to be able to put a measure on a pointless topological space. We will find that there is also a problem in putting a measure on a pointless topological space. I will therefore advocate switching to the measure theoretic approach. But first some of the details of the topological approach.

Let us start with an ordinary pointy topological space which is a 'locally compact $\mathrm{T}_{2}$ space'. A topological space is a ' $\mathrm{T}_{2}$ space' if and only if for any distinct points $x$ and $x$ ' there are disjoint open subsets $O$ and $O^{\prime}$ ' containing $x$ and $x^{\prime}$ respectively. This is a very mild separability condition. A topological space is 'locally compact' if and only if for every point $x$ there exists a 'compact' closed set $C$ such that $x$ lies in the 'interior' of $C$. A set $S$ is 'compact' if and only if 
for every collection of open sets $\left\{O_{\mathrm{a}}\right\}$ such that $S$ is a subset of the union of these open sets, $S \subset \mathrm{U}\left\{O_{\mathrm{a}}\right\}$, there is a finite subcollection of these open sets such that $S$ is a subset of the union of that subcollection: $S \subset O_{\mathrm{a} 1} \mathrm{U} O_{\mathrm{a} 2} \mathrm{U} \ldots . . . \mathrm{U} O_{\mathrm{an}}$. The demand that a space be locally compact is very mild and roughly speaking amounts to the demand that each point is contained in an open set whose closure is not 'very large'. (The closure of a set $S$ is the union of $S$ with its boundary.)

Now let us put on our blurry spectacles, and ignore differences between sets that 'differ only on their boundaries'. We will say that two sets $A$ and $B$ 'differ only on their boundaries' if and only if the closure of the interior of $A$ is equal to the closure of the interior of $B$, i.e. if $\operatorname{ClInt}(A)=\operatorname{ClInt}(B)$. (The interior of a set consists of the points of that set that do not lie on its boundary.) Here are a couple of examples of sets that, by this definition, differ only on their boundaries. Any set and its interior differ only on their boundaries. $(\operatorname{ClIntInt}(A)=\operatorname{ClInt}(A)$ ) $)$ Any set consisting of finitely many points and any other set consisting only of finitely many points differ only on their boundaries, since the closure of the interior of each of them is the empty set.

Now, let us divide up all pointy regions (all sets of points) into equivalence classes $\mathrm{R}$ of regions that differ only on their boundaries. The motivation for doing this is that our 'blurry glasses' can not distinguish regions that are in the same equivalence class, so we can regard these equivalence classes as corresponding to pointless regions. (From here on the symbols ' $R$ ' and ' $\mathrm{R}_{\mathrm{i}}$ ' will be always taken to denote pointless regions rather than pointy regions.)

Now let us give these equivalence classes R mereological structure. (This mereology will be standard except that it will include a 'null region', i.e. it will be a complete Boolean algebra.) In order to do this, let me first note that every equivalence class of pointy regions will include exactly one 'regular closed' pointy region, where pointy region $S$ is said to be 'regular closed' if and only if $\operatorname{ClInt}(S)=S$. For, take pointy region $S$ in some equivalence class. Now consider ClInt $(S)$. It will be in the same equivalence class as $S$, since $\operatorname{ClInt}(S)=\operatorname{ClIntClInt}(S)$. For the same reason CIInt $(S)$ is regular closed. It is also the only regular closed pointy region in that equivalence class. For suppose $S^{\prime}$ is regular closed and in the same equivalence class as $S$. Then $\operatorname{ClInt}(S)=\operatorname{ClInt}\left(S^{\prime}\right)=S^{\prime}$, so $S^{\prime}$ is the same as $\operatorname{ClInt}(S)$. So there is a 1-1 correspondence between pointless regions R, and regular closed pointy regions PR. So we can define a mereological structure on the equivalence classes R by defining a mereological (Boolean) structure $(\leq, \neg, \wedge$, $\checkmark)$ on the corresponding regular closed pointy regions PR. This we can define in the following 
way:

1) The empty set is the null region

2) $\mathrm{PR}_{\mathrm{i}} \leq \mathrm{PR}_{\mathrm{j}}$ if and only if $\mathrm{PR}_{\mathrm{i}} \subset \mathrm{PR}_{\mathrm{j}}$. (' $\leq$ ' stands for the 'part of' relation.)

3) $\neg \mathrm{PR}=\mathrm{Cl}(\mathrm{Co}(\mathrm{PR})) .(\mathrm{Co}(\mathrm{PR})$ is the set theoretic complement of $\mathrm{PR}$.)

4) $P_{\mathrm{i}} \vee P R_{\mathrm{j}}=\mathrm{PR}_{\mathrm{i}} \cup P R_{\mathrm{j}}$

5) $\mathrm{PR}_{\mathrm{i}} \wedge \mathrm{PR}_{\mathrm{j}}=\mathrm{Cl}\left(\operatorname{IntPR}_{\mathrm{i}} \cap \operatorname{IntPR}_{\mathrm{j}}\right)$

6) If $S$ is a set of regular closed pointy regions then $\vee S=C l \cup\{P R \mid P R \in S\}$

7) If $S$ is a set of regular closed pointy regions then $\wedge S=C I I n \cap\{P R \mid P R \in S\}$.

Next let us give the pointless regions topological structure. The topological structure we will give pointless regions can not be given in the same way that we gave pointy spaces topological structure, namely in terms of a distinction between open and closed regions. For that is exactly the kind of distinction that we do not believe exists if reality is pointless. Instead we will give the topological structure of pointless regions in terms of the primitive notions of 'part of', 'connectedness' and 'limitedness'. And again, we will use the 1-1 correspondence with regular closed pointy regions to determine the topological structure of the pointless regions. In particular, we stipulate that

1) Two pointless regions are 'connected' if and only if the closed regular pointy regions that they correspond to have non-empty intersection,

2) A pointless region is 'limited' if and only if the closed regular pointy region that it corresponds to is compact.

Now we can make use of a result that Peter Roeper proved (in Roeper 1997). He has shown that any collection of pointless regions that is constructed in the above way (i.e. by taking equivalence classes of pointy regions in a locally compact $T_{2}$ space which differ only on their boundaries) will satisfy the following axioms of pointless topology:

$\mathrm{A}_{1}$ If pointless region $\mathrm{A}$ is connected to pointless region $\mathrm{B}$, then $\mathrm{B}$ is connected to $\mathrm{A}$ $\mathrm{A}_{2}$ Every pointless region that is not the pointless 'null region' is connected to itself. (The pointless 'null region' corresponds to the equivalence class of regions which differ only 
on their boundaries from the null set.)

$\mathrm{A}_{3}$ The null region is not connected to any pointless region

$\mathrm{A}_{4}$ If $\mathrm{A}$ is connected to $\mathrm{B}$ and $\mathrm{B}$ is a part of $\mathrm{C}$ then $\mathrm{A}$ is connected to $\mathrm{C}$

$\mathrm{A}_{5}$ If $\mathrm{A}$ is connected to the 'fusion' of $\mathrm{B}$ and $\mathrm{C}$, then $\mathrm{A}$ is connected to $\mathrm{B}$ or $\mathrm{A}$ is connected to $\mathrm{C}$.

(The 'fusion' of $\mathrm{B}$ and $\mathrm{C}$ is the smallest pointless region that has $\mathrm{B}$ and $\mathrm{C}$ as parts.)

$\mathrm{A}_{6}$ The null region is limited

$\mathrm{A}_{7}$ If $\mathrm{A}$ is limited and $\mathrm{B}$ is a part of $\mathrm{A}$ then $\mathrm{B}$ is limited

$\mathrm{A}_{8}$ If $\mathrm{A}$ and $\mathrm{B}$ are limited then the fusion of $\mathrm{A}$ and $\mathrm{B}$ is limited

$\mathrm{A}_{9}$ If $\mathrm{A}$ is connected to $\mathrm{B}$ then there is a pointless limited region $\mathrm{C}$ such that $\mathrm{C}$ is a part of $\mathrm{B}$, and $\mathrm{A}$ is connected to $\mathrm{C}$

$\mathrm{A}_{10}$ If $\mathrm{A}$ is limited, $\mathrm{B}$ is not the pointless null region, and $\mathrm{A}$ is not connected to the 'complement' of $\mathrm{B}$, then there is a pointless region $\mathrm{C}$ which is non-null and limited, such that $\mathrm{A}$ is not connected to the 'complement' of $\mathrm{C}$, and $\mathrm{C}$ is not connected to the 'complement' of $\mathrm{B}$. (The 'complement' of a pointless region $\mathrm{A}$ is the pointless region - $\mathrm{A}$ such that $\mathrm{A}$ and -A have no parts in common, and every non-null pointless region has some part in common either with A or with -A.)

From a philosophical point of view it might seem that it would have made more sense to start with the axioms of pointless topology, and then to explain that any collection of pointless regions which satisfies these axioms will correspond 1-1 to equivalence classes of pointy regions in the unique corresponding locally compact $\mathrm{T}_{2}$ space. After all, I certainly do not want to say that pointless regions just are equivalence classes of pointy regions, for that would mean that pointless regions just are mathematical constructions out of entities (pointy regions) which I believe not to exist. And that would not make much sense. No, the pointless view that I am here exploring is that pointy regions really do not exist, let alone that equivalence classes of them exist. The things that really exist are pointless regions, the primitive predicates and relations that are needed are the 'part of' relation, the 'limitedness' predicate and the 'connected to' relation, and the axioms that characterize the true topology of space are $\mathrm{A}_{1}$ through $\mathrm{A}_{10}$. However, not only is it much easier to introduce the machinery of pointless topologies via a construction out of pointy topologies, it is also very important to see that pointless regions behave exactly the way 
that our blurry spectacle motivation wants them to behave. That is why I constructed pointless topologies in the way that I did. OK, on to the next tasks: placing material objects and fields in such a pointless topological space, and giving this space more structure than topological structure.

\section{Objects in a pointless topology}

If space is pointless then one can not specify the locations of material objects by indicating for each point in space whether that object occupies it or not. So how should we conceive of the locational properties of objects in a pointless space? Well, here's a suggestion. We specify the locational state of a material object by specifying for every pointless region whether the object is entirely contained in that region or not.

This suggestion is problematic. The problem is that, despite the fact that space is pointless, one could nonetheless have point particles if one followed this suggestion. How? Well, imagine that a material object is such that it is entirely contained in each of a collection of smaller and smaller pointless regions. Now, if for any pointless region within which the object is contained there is an arbitrarily small pointless subregion within which the object is contained, the object could not have any finite size. So it must have size zero. ${ }^{4}$ This is surprising. For it means that one can have pointless space containing point particles! However, allowing such a thing seems to defeat most of the reasons we started on this whole business of gunk. We wanted neither points in space nor point particles.

Moreover, allowing such point particles also leads to a formal feature that seems objectionable, namely a violation of 'countable additivity'. Here's what that means in this context and why it fails. Consider the following plausible looking principle: if an object is wholly outside each of a countable collection of regions $R_{i}$, then it is also wholly outside the fusion of these regions. Now consider our example. If a particle is entirely contained in each of a collection of converging regions, then it is wholly outside the complements of these regions.

4 This doesn't quite mean that it as to be a point particle, since it could still be a line, or an infinitely thin surface. But one can define a notion of 'a converging set of regions' in such a way that the particle does indeed have to be a point particle if it is entirely within each of the regions in the 'converging set of regions'. 
Now consider the fusion of the complements of these regions. Intuitively speaking the only thing that this fusion does not contain is the point that the converging collection is converging to. But remember that we are in a pointless space, and there exists no such point. So one should expect that the fusion of this countable collection is the whole space, for there is no pointless region that it misses out on, as it were. And indeed, this is correct: the fusion of these complements is the whole space. But a material object can not lie entirely outside the whole of space. So we have a countable collection of regions such that the object lies wholly outside each one of the regions in the collection, but is wholly contained in their fusion. This is a failure of countable additivity, and seems bizarre and objectionable. So it seems that one should not allow a specification of the locational properties of a material object by specifying for each region whether it is entirely contained in it or not.

The obvious alternative is the following. One specifies the locational properties of a material object by specifying which region the object exactly fills. It will then, of course, be entirely contained in any region that includes this region, etc. But it could not be entirely contained in a converging collection of regions, for there is a minimal region, such that it is not contained in anything smaller than that region. No problem.

\section{Fields in a pointless topology}

How about the states of a field such as the electric field in a pointless topology? Here's a very natural suggestion. We specify the state of a field by specifying for each pointless region in space the exact range of values that the field obtains in that region. This brings up a further issue. Should we think of the possible ranges of values of the field as pointless ranges or as pointy ranges? Should we think that fields can have exact point values, or that the value spaces of fields are as gunky as the physical space that they inhabit? I don't know. In what follows I will make the weaker assumption, i.e. I will assume that the value space of a field is a pointless space, and see how far we get.

Following Peter Roeper (Roeper 1997), let us call a map h from a pointless physical space $\mathrm{S}$ to a pointless field value space VS a 'bounded continuous mereological' map, if it satisfies the following constraints:

(1) $h(R)$ is the null region in VS if and only if R is the null region in S 
(2) If $R_{1}$ is part of $R_{2}$ then $h\left(R_{1}\right)$ is part of $h\left(R_{2}\right)$

(3) If $V$ is non-null and part of $h\left(R_{1}\right)$ then there exists a non-null $R_{2}$ such that $R_{2}$ is part of $R_{1}$ and $h\left(R_{1}\right)$ is part of $V$

(4) If $R_{1}$ is connected to $R_{2}$ then $h\left(R_{1}\right)$ is connected to $h\left(R_{2}\right)$

(5) If $\mathrm{R}$ is limited, then $\mathrm{h}(\mathrm{R})$ is limited.

One can prove (see Roeper 1997) that there is a 1-1 correspondence between bounded continuous mereological maps (between two pointless spaces) and continuous pointy functions (between the two corresponding locally compact $\mathrm{T}_{2}$ spaces) which map regular closed sets of points to regular closed sets of points. That is to say, if we specify the state of a pointless field in a pointless space by means of a bounded continuous mereological map $\mathrm{h}$, then this is equivalent to specifying the corresponding pointy field in the corresponding pointy space by means of a function $\mathrm{f}$ from points in space to pointy field values, where $\mathrm{f}$ must satisfy the constraint that it maps regular closed sets of points in space to regular closed sets of field values.

Now, suppose that it were the case that every pointy field function $\mathrm{f}$ that one ever is likely to need when doing standard pointy physics has the feature that it sends regular closed sets to regular closed sets. Then one could suggest that even though physical space and field value spaces in fact are pointless, one can still continue the standard practice of using ordinary pointy functions $\mathrm{f}$ when doing one's calculations in physics, since the possible gunky field states in gunky space correspond 1-1 to such pointy functions in pointy space.

Unfortunately, though, this is not true. For consider a pointy function $\mathrm{f}$ that has a fixed constant value $\mathrm{v}$ over some pointy region PR. It will map every subset of PR, and hence every regular closed subset of PR, to the singleton set $\{\mathrm{v}\}$. And a singleton is not a regular closed set. So a function that is constant over some (finite) region PR does not preserve the property of being regular closed. But clearly physics needs to make use of such functions. So we have a problem.

And there is more trouble. It seems clear that we will need to put a measure on pointless regions. For how else are we going to able to talk of the sizes of regions, and how else are we going to be able to do the pointless analogue of the integration of functions, something that we surely have to be able to do. Unfortunately when one tries to put a measure on a pointless topological space one will run into difficulties that appear to be insurmountable. 
Let me start on the project of putting a measure on a pointless topological space by considering a very simple case. We know that there is a 1-1 correspondence between pointless topological spaces and pointy locally compact $\mathrm{T}_{2}$ spaces. Let us now consider the pointless topological space that corresponds to the pointy 1-dimensional continuum, i.e. the real number line. We know that there is a 1-1 correspondence between the pointless regions $\mathrm{R}$ in the pointless 1-dimensional continuum and the regular closed sets of real numbers. Given this fact, the obvious way to try to put a measure on the pointless regions in the pointless continuum is to identify the measure of any pointless region $\mathrm{R}$ with the Lebesque measure of the corresponding closed regular set of real numbers PR. The problem now is that this will turn out to yield a measure on the pointless regions which violates countable additivity. We can see this by looking at a 'Cantor-set', or rather, the complement of a Cantor set.

Start with the set $[0,1]$. Call this set $\mathrm{S}_{0}$. It is a regular closed set with Lebesque measure 1. Now consider the middle quarter of this set, i.e. the set $[3 / 8,5 / 8]$. Call this set $S_{1} . S_{1}$ is a regular closed set with Lebesque measure 1/4. Now consider the set $\mathrm{S}_{2}$ which has consists of two parts which fill the middle of the gaps left by $S_{1}$ and which has Lebesque measure 1/8. That is to say $S_{2}=[7 / 32,9 / 32] \cup[23 / 32,25 / 32]$. Keep on doing this. I.e. set $S_{n}$ has parts which are slotted halfway between all the parts of all the previous sets, and $S_{n}$ has half the Lebesque measure of set $S_{n-1}$. Since each set $S_{n}$ is a regular closed set, each such set corresponds to a pointless region $R_{n}$ in the pointless 1-dimensional continuum. Let us now ask what the fusion $\vee\left\{\mathrm{R}_{\mathrm{n}}\right\}$ of all these pointless regions is. Well, by our previous account of the mereology of pointless regions, this is going to be the unique pointless region that corresponds to the regular closed pointy region $C l \cup\left\{\mathrm{S}_{n}\right\}$. The union of all pointy regions $S_{n}$ is dense on the set $[0,1]$. So its closure is just $[0,1]$. So the pointless region $\vee\left\{R_{n}\right\}$ corresponds to the equivalence class of regions that differs by measure 0 from the pointy region $[0,1]$.

Now we can see why we are in trouble if we assign measures to pointless regions by assigning them the Lebesque measure of the unique regular closed regions that they correspond to. For $\vee\left\{R_{n}\right\}$ will be assigned measure 1 by this method, while the measures of the $R_{n}$ will sum to $1 / 2$. That is to say, this measure will not be countably additive. This is a terrible problem, for we need a countably additive measure.

Now one might suggest that the problem here is that I simply suggested the wrong rule 
for assigning measures to pointless regions. However, not only is there no other obvious candidate for such a measure, one can in fact prove that there can be no such measure. That is to say, one can prove that there can not exist a countably additive measure that is defined on every element of an algebra if that algebra is isomorphic to the algebra of closed regular regions of a continuum. ${ }^{5}$ So our attempt to do physics in this kind of pointless topological space is in big trouble. Combined with the implausibility of our account of the possible states of fields in this kind of topological space, this provides us with a good reason to try our luck instead with the measure theoretic approach to pointless spaces.

\section{The measure theoretic approach to pointless spaces}

Let's concentrate on a simple case: the real number line. As before, we are going to create a pointless space by putting on blurry glasses. On the measure theoretic approach what we are going to blur out is differences of Lebesque measure 0 . In order to do that, we first have to restrict ourselves to Lebesque measurable sets. So let's start by restricting ourselves to the Borel sets. One gets the collection of all Borel sets on the real line by starting with the collection of all open intervals (open sets of the form $(a, b)$ for any real numbers $a$ and $b$ ), and closing this collection up under complementation, countable union and countable intersection. Now let us put on our blurry glasses and define pointless regions $\mathrm{R}$ of the pointless real line to be equivalence classes of Borel sets of the pointy real line that differ up to Lebesque measure 0 . Note that forming such equivalence classes preserves complementation, countable union and countable intersection. Indeed one can show that the algebra of such equivalence classes is a complete Boolean algebra, i.e. a standard mereology (with a null region) which is closed under arbitrary fusion. (See Sikorski 1964, pages 73-75.)

As before we would like to be able to recover standard physics, and we would therefore

${ }^{5}$ This is so because the algebra of closed regular regions of the real line is not 'weakly distributive', and one can not have a 'semi-finite' countably additive measure that is defined on every element of an algebra that is not weakly distributive. A measure is said to be 'semi-finite' if every element that has infinite measure has a part that has finite measure. For the definition of weak distributivity and a proof of the fact that one can not put a semi-finite measure on an algebra that is not weakly distributive, see chapters 32 and 33 of Fremlin 2002. 
like to be able to recover a large collection of pointy functions from pointy space to a pointy field value space from some suitable collection of mappings between the corresponding pointless spaces. Luckily there already exists a nice and well-known account of how to do this. In particular, one can prove the following (see Sikorski 1964, section 32). There exists a 1-1 correspondence between equivalence classes of pointy 'Borel-measurable' functions from real line A to real line B that differ on up to Lebesque measure 0 sets of points, and ' $\sigma$ homomorphisms' from pointy regions on the pointy real line B to pointless regions on the pointless real line A. A function is said to be 'Borel measurable' if it sends Borel sets to Borel sets. A mapping $\mathrm{h}$ between Boolean algebras that are closed under countable union and intersection is said to be a ' $\sigma$-homomorphism' if :

1) $h(\neg R)=\neg h(R)$

2) $h\left(V R_{i}\right)=V h\left(R_{i}\right)$, for any countable collection $\left\{R_{i}\right\}$

3) $h\left(\wedge R_{i}\right)=\wedge h\left(R_{i}\right)$, for any countable collection $\left\{R_{i}\right\}$.

That is to say, if we make the very simple and natural assumption that the state of a pointless scalar field in a pointless continuum (the above generalizes to $\mathrm{n}$-dimensional continua) can be given by a $\sigma$-homomorphism from pointy value ranges to pointless regions in space, then we can recover all Borel measurable pointy functions (including highly discontinuous ones) up to differences of Lebesque measure 0 . This is a great result. Not only can one recover all the functions that one could reasonably be expected to ever need in physics, one can also only recover these functions up to the kind of differences that one would expect not to correspond to real differences in nature.

What about topology though? We have just put a measure on an atomless mereology of pointless regions, but that tells us nothing about which pointless region is connected to which pointless region. For, loosely speaking, cutting out a segment of the real line, and pasting it in somewhere else along the real line does not alter the mereology of the real line, nor the measure theoretic structure of that mereology. So we need to add a topology separately. How could we do that? Well, what we could try to do is to start with the pointy real line, and then use its pointy topology to define a topology on the pointy real line which is invariant under differences of up to Lebesque measure 0 . Let's try that.

Let's say that pointy Borel sets A and B are 'connected' if and only if there exists a point 
$p$ such that any open set containing $p$ has an overlap of non-zero measure both with $\mathrm{A}$ and with B. And let us say that pointy Borel set A is 'limited' if and only if for some compact Borel set B we have that $\mathrm{A} \cap$ Complement(B) has measure 0 .

Clearly these definitions are invariant under differences in regions A and B up to measure 0 . So we can use it to define a topology on the pointless regions of the pointless real line. The resulting structure will satisfy Roeper's axioms A1 through A9, but it will violate axiom A10. Let me remind you what this axiom says: If $\mathrm{R}_{\mathrm{a}}$ is limited, $\mathrm{R}_{\mathrm{b}}$ is not the pointless null region, and $R_{a}$ is not connected to the complement of $R_{b}$, then there is a pointless region $R_{c}$ which is non-null and limited, such that $R_{a}$ is not connected to the complement of $R_{c}$, and $R_{c}$ is not connected to the complement of $\mathrm{R}_{\mathrm{b}}$.

To see that this axiom fails consider a Cantor-type pointy set, for instance the pointy set $B=(0,1) \cap$ complement $\left(\cup S_{n}\right)$ where the $S_{n}$ are the gap-filling sets that I defined in the previous section. Set $B$ is a measure $1 / 2$ Borel set, so we can consider the corresponding non-null pointless region $R_{b}$ to which it corresponds. Now let $R_{a}$ be the null region. $R_{a}$ is limited and not connected to the complement of $R_{b}$ since the null region is not connected to anything. So there should be $a$ non-null and limited $R_{c}$ such that $R_{c}$ is not connected to the complement of $R_{b}$. Now the complement of $R_{b}$ is the union of three pointless regions: $\{-\infty,-1\}, \cup R_{n}$, and $\{1, \infty\}$. Now, any pointy non-null open set has an overlap of non-zero Lebesque measure with any pointy set in the equivalence corresponding to this pointless region, so this pointless region is connected to every non-null pointless region. So there can not be such an $R_{c}$.

The problem is the following. The basic idea of axiom A10 is that there is a topological notion of pointless region $R_{1}$ being 'strictly inside' pointless region $R_{2}$. The idea is that $R_{1}$ is strictly inside $R_{2}$ if $R_{1}$ is disconnected from the complement of $R_{2}$. And then the idea of 'pointlessness', or the idea of 'non-atomicity' suggests that if $\mathrm{R}_{1}$ is strictly inside $\mathrm{R}_{3}$ then there ought to be an $R_{2}$ such that $R_{1}$ is strictly inside $R_{2}$ and $R_{2}$ is strictly inside $R_{3}$. In particular for any non-null R there should be a non-null region R' which is strictly inside $\mathrm{R}$. This axiom fails given the way that I have defined connectedness on the measure theoretic approach, since there are Cantor type non-null regions such that there are no regions that are strictly inside such a Cantor type region, since the complement of such a Cantor type region is connected to every non-null region. 
Now one might think that the failure of this axiom shows that we do not really have a pointless space. However, the fact that our space is pointless is still unambiguously represented in two different ways:

1) The algebra of regions is non-atomic

2) Other than the null region, every region has non-zero measure, and for every non-zero measure, no matter how small, there are regions that have that measure.

So I am not terribly worried about the failure of axiom A10. However, it is interesting to note that the fact that there exists a pointless region $\mathrm{R}_{\mathrm{b}}$ corresponding to a pointy Cantor set shows that one should not think that each pointless region can be decomposed into a collection of extended 'solid islands'. The pointless region $\mathrm{R}_{\mathrm{b}}$, for instance, is not so decomposable. Ah well, so be it.

There is a question that I have not yet answered. Namely: to what extent does a measure algebra with a topology satisfying axioms A1-A9 uniquely correspond to a pointy topological space plus measure? Part of the answer is well known: every atomless separable measure algebra is isomorphic, and hence corresponds uniquely to the mereology of the continuum with the Lebesque measure on its Borel algebra. (See Royden 1968, chapter 15.) But I do not yet know to what extent the pointless topological structure uniquely determines the corresponding pointy topological structure. So there is interesting work left.

And, of course, this is only a beginning. We also need to add differential structure and then metric structure in order to be able to do modern physics. But that is work for the future. For now let me simply conclude that the measure theoretic approach to gunky, or pointless, spaces is the most promising. 


\section{REFERENCES}

Arntzenius , F. (2000) “Are there really instantaneous velocities?”, The Monist Vol 83 No 2, pp $187-208$.

Arntzenius, F. (2004): “Is quantum mechanics pointless?”, Philosophy of Science Vol 70 No 5, pp 1447-1458.

Böhm, A. (1978): The Rigged Hilbert Space and Quantum Mechanics. Berlin: Springer.

Fremlin, T. (2002): Measure Theory, Vol 3. Published by Fremlin himself. Available at http://www.essex.ac.uk/maths/staff/fremlin/mtsales.htm.

Gerla, G. (1990): "Pointless metric spaces", The Journal of Symbolic Logic Vol 55 No 1, pp 207219.

Halvorson, H. (2001): "On the nature of continuous physical quantities in classical and quantum mechanics", Journal of Philosophical Logic Vol 30, pp 27-50

Roeper, P. (1997): “Region-based topology”, Journal of Philosophical Logic Vol 26, pp 251309.

Royden, H. (1968): Real Analysis. London: MacMillan.

Sikorski, R. (1964): Boolean Algebras. Berlin: Springer Verlag.

Skyrms, B. (1983): “Zeno's paradox of measure”, in Cohen, R.S. and Laudan, L. (eds., 1983): Physics, Philosophy and Psychoanalysis, pp 223-254.

Skyrms, B. (1993): "Logical atoms and combinatorial possibility", The Journal of Philosophy Vol 90 No 5, pp 219-232.

Wagon, S. (1985): The Banach-Tarski Paradox. Cambridge: Cambridge University Press. 